\title{
Risk Factors and Posttraumatic Stress Disorder: Are They Especially Predictive Following Exposure to Less Severe Stressors?
}

\section{Citation}

McNally, Richard J., and Donald John Robinaugh. 2011. Risk factors and posttraumatic stress disorder: Are they especially predictive following exposure to less severe stressors? Depression and Anxiety 28(12): 1091-1096.

\section{Published Version}

doi:10.1002/da.20867

\section{Permanent link}

http://nrs.harvard.edu/urn-3:HUL.InstRepos:8916390

\section{Terms of Use}

This article was downloaded from Harvard University's DASH repository, and is made available under the terms and conditions applicable to Open Access Policy Articles, as set forth at http:// nrs.harvard.edu/urn-3:HUL.InstRepos:dash.current.terms-of-use\#OAP

\section{Share Your Story}

The Harvard community has made this article openly available.

Please share how this access benefits you. Submit a story.

\section{Accessibility}


Running head: TESTING CONCEPTUAL BRACKET CREEP

\title{
Depression and Anxiety (in press)
}

\author{
Risk Factors and Posttraumatic Stress Disorder: \\ Are They Especially Predictive Following Exposure \\ to Less Severe Stressors? \\ Richard J. McNally and Donald J. Robinaugh \\ Harvard University \\ Author Note
}

Richard J. McNally and Donald J. Robinaugh, Department of

$$
\text { Psychology, Harvard University }
$$

This research was supported by a grant from the Milton Fund, Harvard University.

We thank Hannah E. Reese and Anne-Laura van Harmelen who tested subjects in the risk and resilience project, and Naomi Breslau whose observations motivated this study and who made comments on our manuscript.

Neither author has financial disclosures or conflicts of interest to report.

Correspondence concerning this article should be addressed to Richard J. McNally, Department of Psychology, Harvard University, 33 Kirkland Street, Cambridge, MA 02138. e-mail: rjmewjh.harvard.edu 
Abstract

The diagnosis of posttraumatic stress disorder (PTSD) requires exposure to a traumatic stressor, as defined by Criterion A in the DSM criteria for PTSD. Yet, over the course of successive revisions of the diagnostic manual the range of qualifying stressors has expanded considerably (e.g., witnessing terrorist attacks on television). Moreover, stressors that still fall short of qualifying for Criterion A can produce apparent PTSD. Taken together, these findings imply that people who do satisfy symptomatic criteria for PTSD following exposure to less severe stressors carry a heavy burden of risk factors. To test this hypothesis, we examined whether the association between the risk factor of lower intelligence and more severe PTSD and depression symptoms would be greater among women reporting less severe childhood sexual abuse (CSA; $n=15)$ relative to women who reported moderate $(n=54)$ or high $(n=31)$ severity CSA. The evidence was consistent with this hypothesis for subjects in the low and moderate severity groups, but less so for those in the high severity group. Keywords: PTSD, sexual abuse, risk factors, resilience, conceptual bracket creep, Criterion A. 
Risk Factors and Posttraumatic Stress Disorder:

Are They Especially Predictive Following Exposure

to Less Severe Stressors?

To receive a diagnosis of posttraumatic stress disorder (PTSD), an individual must first meet Criterion A: exposure to a traumatic event. ${ }^{[1]}$ The assumption is that only events that qualify for this definition of trauma possess the capacity to produce the symptomatic profile of PTSD.

However, since the appearance of PTSD in the third edition of the Diagnostic and Statistical Manual (DSM-III) ${ }^{[2],}$ there has been a considerable expansion in the types of events that meet Criterion A. Because of this conceptual bracket creep in the definition of trauma, [3] events that never would have qualified according to DSM-III now qualify according to DSM-IV-TR. ${ }^{[1]}$ For example, the current edition of the diagnostic manual no longer requires that someone be physically present at the scene of the trauma to qualify as a trauma survivor. ${ }^{[4]}$ That is, people who feel helpless when learning about threats to the safety of others are victims of trauma just as much as those whose lives were in danger. Indeed, researchers have reported that 4\% of Americans living far from the sites of the terrorist attacks of september 11, 2001 developed presumptive PTSD, ${ }^{[5]}$ apparently by viewing 
television coverage of the violence. Being physically present at the scenes of the attacks would always have qualified as exposure to a catastrophic stressor, but merely watching the events unfold on television would not, according to the original DSM-III concept of trauma.

In parallel with the expansion in the DSM definition of trauma, researchers have examined whether stressors that fall short of Criterion A may nevertheless result in PTSD. As Dohrenwend $^{[6]}$ recently observed, at least 12 studies have shown that these non-Criterion A stressors are often associated with PTSD symptoms or with the full syndrome itself. In some studies, non-Criterion A events were associated with greater severity of PTSD symptoms than were Criterion A events. For example, college students whose worst life event did not qualify for Criterion A reported more PTSD symptoms than did college students whose worst life event met Criterion A [7, 8]. Similarly, a survey of Dutch women randomly sampled from the community revealed that for adverse events from the previous 30 years, stressors that fell short of Criterion A were associated with higher PTSD scores than were stressors that qualified for Criterion A [9]. A survey of Australians likewise revealed that rates of PTSD were higher for non-Criterion A stressors than for Criterion 
A stressors [10]. A study of American women interviewed about their worst life events indicated that directly experienced non-Criterion A events produced PTSD significantly more often than did directly experienced Criterion A events. [11] Taken together, these studies seemingly impugn the DSM assumption that only Criterion A events possess the capacity to produce PTSD .

What should we make of these findings? One interpretation is that symptom self-reports of college students or community subjects do not signify "real" PTSD as diagnosable via clinical interview. That is, these subjects may misunderstand the meaning of emotional numbing, flashbacks, and so forth, confusing normal reactions with psychopathology. Questionnaires can overestimate clinically diagnosable PTSD, even among war veterans ${ }^{[12]}$.

Another interpretation is that symptoms signify genuine PTSD, but that its source originates at least as much in subjects' preexisting vulnerabilities as in the nominal stressor itself. In fact, the majority of people who encounter trauma do not develop PTSD, [13] indicating that victims vary in their vulnerability for the disorder. Accordingly, to elucidate the etiology of PTSD, clinical scientists have endeavored to identify variables that either 
heighten or diminish risk for PTSD among victims of trauma. [14] For example, studying Vietnam veterans, McNally and Shin ${ }^{[15]}$ found that lower levels of intelligence predicted greater PTSD symptom severity, even when they controlled statistically for extent of combat exposure and level of education. Prospective longitudinal studies have confirmed that pretrauma intelligence predicts PTSD symptoms or diagnosis with higher levels of intelligence conferring protection against PTSD among Vietnam combat veterans, [16] Israeli combat veterans, ${ }^{[17]}$ and American children exposed to civilian trauma. ${ }^{[18]}$

It makes intuitive sense that someone whose PTSD occurs after exposure to a low-severity stressor qualifying for Criterion A under bracket creep (e.g., viewing news coverage of 9/11) or to a non-Criterion A stressor must carry a heavy burden of vulnerability to fall ill. That is, a backgroundforeground inversion may occur whereby the importance of the stressor recedes into the causal background as the vulnerability factors move into the causal foreground for people who meet PTSD criteria after experiencing a lowmagnitude stressor. ${ }^{[19]}$ If so, then the strength of association between a risk factor and PTSD symptoms should be much greater for those who have encountered relatively low- 
magnitude stressors than for those who have encountered highmagnitude stressors.

Yet, as Breslau ${ }^{[20]}$ observed, no one has directly tested this hypothesis. Indeed, it seems self-evidently true. However, some indirect evidence suggests that it may be incorrect. Noting the apparent absence of studies on the relative impact of risk factors on PTSD among people exposed to stressors of varying severity, Breslau[20] discussed Helzer's ${ }^{[21]}$ early study on depression in Vietnam veterans. Helzer identified premilitary variables, such as childhood antisocial behavior, drug use, and failure to graduate from high school that predicted postmilitary depression. Contrary to his expectation, he found that the effect of predisposition was greatest in veterans with the most trauma exposure. For example, a premilitary history of drug use dramatically increased the risk of depression among wounded versus nonwounded veterans. As Helzer ${ }^{[21]}$ observed, such "findings are inconsistent with the hypothesis that antecedents have a lesser predictive effect under conditions of extreme stress" (p. 121).

Interpersonal traumatic events, such as rape, result in PTSD far more often than do impersonal traumatic events, such as motor vehicle accidents. ${ }^{[22]}$ Accordingly, reasoned 
Breslau, [20] the effect size for the association between a risk factor and PTSD should be greater for impersonal trauma than for interpersonal trauma. Yet contrary to this prediction, she observed that the effect sizes for the association between risk factors and PTSD was greater for interpersonal trauma than for impersonal trauma, specifically, motor vehicle accidents. ${ }^{[23]}$

Inspired by Breslau's ${ }^{[20]}$ observations, we directly tested the background-foreground inversion hypothesis ${ }^{[19]}$ by examining the association of intelligence to PTSD and depression symptoms at varying levels of stressor severity in women who report a history of childhood sexual abuse (CSA). To evaluate whether the magnitude of the association between intelligence and symptoms of PTSD and depression varied as a function of stressor severity, we classified CSA subjects as experiencing low, medium, or high CSA severity. We predicted that the strength of association between intelligence and symptoms would be stronger in the low severity group than in the medium severity group, and weakest in the high severity group. That is, as the magnitude of the stressor declines, the effect of the risk factor on PTSD and depression symptoms should increase.

Method 


\section{Subjects}

The subjects were 102 women who had responded to our newspaper announcement inviting women to participate in a research project concerning risk and resilience variables in women who had experienced sexual abuse during childhood. A brief interview during the phone screen determined eligibility (i.e., that the subject recalled at least one episode of sexual abuse involving physical contact comprising fondling, oral-genital contact, or sexual intercourse).

\section{Procedure}

Upon arrival at our laboratory, subjects read and signed an informed consent form that described the study. The Institutional Review Board of Harvard University approved the protocol and consent form. Subjects provided a saliva sample for genotyping and participated in two cognitive tasks unrelated to the hypotheses tested in this article. Subjects then completed a battery of questionnaires, including assessments of post-trauma psychopathology, characteristics of the trauma memory, and a questionnaire about their recollections of abuse. ${ }^{[24]}$

We constructed a measure of trauma severity by scoring two open-ended questions from the abuse recollections questionnaire, one asking about the perpetrator and the other 
asking about the number of episodes of CSA. We classified our subjects into three categories of trauma severity. The lowest level included those subjects who reported only a single incident of CSA by a perpetrator who was not a nuclear family member (e.g., a stranger or an older cousin). The medium level of severity included those subjects who experienced a single incident by a nuclear family member (e.g., a father or an older brother) or experienced more than one incident by a perpetrator who was not a nuclear family member. The highest level included those subjects who had experienced more than one incident by a perpetrator who was a nuclear family member. Scores on the severity measure ranged from two to four. We predicted that the strength of association between intelligence and PTSD symptoms and depression symptoms would be greatest for those subjects in the lowest level of stressor severity.

The low severity group included 15 women, the medium severity group included 54 women, and high severity group included 31 women. We lacked severity data on two subjects (e.g., subject declined to note whether the perpetrator was a nuclear family member) whose other data we included for the overall analyses testing the correlation between intelligence and PTSD and depression symptoms, respectively. 


\section{Measures}

Posttraumatic Checklist, Civilian version (PCL-C). ${ }^{[25]}$ One of the most widely used self-report measures of PTSD, ${ }^{[26]}$ the PCL-C contains 17 items that constitute the symptomatic criteria for PTSD. Subjects rate each item on a five-point scale ranging from one (Not at all) to five (Extremely) to indicate how seriously the symptom has bothered them during the past month. Total scores can range from 17 to 85 . Subjects rate each item in reference to a "stressful life experience" (e.g., childhood sexual abuse). Following usual practice, we summed the scores for each of the 17 items, thereby obtaining a dimensional measure of posttraumatic symptoms. We also identified subjects whose total score was 50 or higher and who met symptomatic criteria for PTSD by scoring at least a three (Moderately) on at least one $B$ (reexperiencing) symptom, at least three (avoidance and numbing) symptoms, and at least two (arousal) symptoms.

Beck Depression Inventory - II (BDI-II). ${ }^{[27]}$ The BDI-II is a 21-item questionnaire that measures symptoms of depression. Subjects rate each item on a severity scale ranging from 0 to 3. Total scores range from zero to 63 .

Shipley Institute for Living Scale. ${ }^{[28]}$ The Shipley scale has a verbal subtest and a nonverbal reasoning subtest. It 
measures cognitive ability, and total scores correlate strongly with full scale WAIS-R IQ $(r=.87) .{ }^{[29]}$ We converted each subject's score into its estimated WAIS-R equivalent.

\section{Results}

Because we had directional predictions, our tests are one-tailed unless otherwise noted. Degrees of freedom vary because of missing data for some subjects.

\section{Preliminary Analyses}

The means and standard deviations for age, IQ, PTSD symptoms, and depression symptoms for the stressor severity groups appear in Table 1. Analyses of variance revealed no significant differences among the groups on these variables. However, the mean age of the high severity group tended to be greater than the mean age of the low severity group.

In the low, medium, and high severity groups, 27\%, 33\%, and $62 \%$ met criteria for presumptive PTSD, respectively. Consistent with the dose-response concept, ${ }^{[30]}$ as trauma severity increased, so did the severity of PTSD symptoms, $r(92)=.21, p=.02$. However, depression severity did not increase significantly as a function of trauma severity, $r(97)=.13, p=.10$.

Replicating previous findings, lower intelligence predicted more severe PTSD symptoms, $r(92)=-.22, p=.02$. 
Intelligence also predicted depression symptoms, r(99) = $.19, p=.03$.

We next examined whether the strength of association between lower intelligence and greater PTSD and depression symptoms varied as a function of stressor severity. Among low stressor severity subjects $(n=15)$, intelligence predicted both severity of PTSD symptoms, $r(13)=-.43, p=$ .05 , and depression symptoms, $r(13)=-.61, p=.008$. The point biserial correlation between intelligence and PTSD caseness was large, $r_{p b}(13)=-.68, p=.002$.

Among medium stressor severity subjects $(n=54)$, intelligence did not significantly predict severity of PTSD symptoms, $r(46)=-.15, p=.15$, nor severity of depression symptoms, $r(51)=-.03, p=.81$. The point biserial correlation between intelligence and PTSD caseness was negligible, $r_{p b}(47)=-.001, p=.50$.

To test whether the effect sizes in the low stressor severity group were larger than were those in the medium stressor severity group, we first converted the correlations to Fisher Z scores. The correlation between intelligence and depression symptoms was significantly greater in the low stressor severity group than in the medium stressor severity group, $z=2.12, p=.02$. The correlation between 
intelligence and PTSD symptoms was not significantly greater in the low stressor severity group than in the medium stressor severity group, $Z=.97, p=.17$. However, the point biserial correlation between intelligence and PTSD caseness was significantly greater in the low stressor severity group than in the medium stressor severity group, $z$ $=2.59, p=.005$. Taken together, these data are generally consistent with the hypothesis that vulnerability factors have stronger association with PTSD (and depression) symptoms in victims whose trauma severity is less marked.

However, the data from our high stressor severity subjects $(n=31)$ were not entirely consistent with this hypothesis. Although the negative association between intelligence and PTSD symptoms for these subjects, $r(28)=-$ .29, $p=.06$, was numerically smaller than for the low stressor severity subjects, it was numerically higher than for the medium stressor severity subjects. A similar pattern held for the intelligence-depression association, $r(29)=-$ $.28, p=.06$. For the high severity group, the point biserial correlation between intelligence and PTSD caseness was nonsignificant, $r_{p b}(27)=-.29, p=.07$. However, consistent with our hypothesis, this correlation was 
marginally less than in the low stressor severity group, $Z=$ $1.51, p=.07$

None of the other differences among effect sizes between stressor severity groups was significant (ps >.05, onetailed)

Discussion

Conceptual bracket creep in the definition of trauma greatly expands the range of stressors that satisfy Criterion Al for PTSD. Although certain stressors, especially interpersonal ones such as rape, are especially likely to result in PTSD, merely feeling helpless upon hearing the news of another person's misfortune can certify recipients as trauma survivors themselves, enabling them to qualify for the diagnosis. Moreover, several studies show that people often report greater PTSD symptom severity following non-Criterion A stressors than following Criterion A stressors.

For those who develop PTSD following low-magnitude stressors, one might expect a background-foreground inversion whereby the stressor recedes into the causal background while risk factors move into the causal foreground. This should hold for both non-Criterion A events and for Criterion A events of relatively low magnitude. Accordingly, the magnitude of the relationship between a risk factor and PTSD 
symptoms should be greater among people exposed to relatively less severe stressors than among people exposed to more severe ones.

Consistent with this hypothesis, for women exposed to presumably less severe CSA, the negative correlation between intelligence and both PTSD and depression symptoms was numerically greater than for women exposed to CSA of moderate severity. The point-biserial correlational data for intelligence and presumptive PTSD-caseness provided the strongest support for our hypothesis. The correlation between intelligence and presumptive PTSD caseness was strongly negative for subjects in the low severity group, essentially zero in the medium severity group, and nonsignificantly negative in the high severity group.

Our study has limitations. First, the severity measure was less than ideal. Because questions about the perpetrator and the frequency of abuse episodes were open-ended, the data sometimes lacked precision. For example, some subjects who reported more than one episode of abuse mentioned that it occurred many times without specifying a number. Accordingly, we grouped subjects who mentioned that it occurred twice with those who said it occurred countless times. Moreover, we assumed that one episode of abuse by a 
nuclear family member was equivalently severe as multiple episodes by non-nuclear family member. This assumption is subject to debate. For example, a single violent sexual assault by a stranger may be more toxic than two episodes of fondling by one's father. Indeed, according to our classification system, an eleven-year-old girl who experienced a brutal rape by a stranger would fall into the low stressor category, thereby adding statistical noise to our analyses.

Nevertheless, despite the limitations of this measure of stressor severity, we still found a dose-response effect with the study group as a whole. This finding provides support for our method of classifying stressful CSA experiences, its limitations notwithstanding. Furthermore, our measures of cognitive ability and PTSD symptoms were reasonably good ones. The Shipley Institute of Living Scale and the PCL-C correlate well with the WAIS-R and structured interviews for PTSD, respectively.

Another limitation is the relatively small size of our study group, especially those in the low severity subgroup. Despite the limited statistical power to detect significant effects, we still corroborated the hypothesis in this group. 
However, the results require cautious interpretation because effects involving small samples are often unstable.

A major purpose of our research was to stimulate research on the background-foreground inversion hypothesis. One might assume that it would be difficult to test the hypothesis that people whose PTSD results from exposure to low magnitude stressors carry a burden of vulnerability. That is, the dose-response principle implies that low magnitude stressors will seldom produce PTSD. Yet studies on non-Criterion A events produce presumptive PTSD far more often than most experts would have predicted.

There are several ways to test the background-foreground inversion hypothesis. One can assess it across stressor categories (e.g., natural disaster versus rape). As we noted previously, interpersonal traumatic events result in PTSD far more often than do impersonal ones. Accordingly, the background-foreground inversion hypothesis would predict that an individual who develops PTSD following an impersonal traumatic event should carry a greater burden of vulnerability than an individual who develops PTSD following an interpersonal traumatic event. [cf. 18]

In addition, one can assess stressor severity within a stressor category, as we did in this study. Within-category 
variation might include whether one directly experienced the event, witnessed it personally, or only learned about it later. Another variable might be extent of injury. Suffice it to say, the prediction would be that the magnitude of effect between a risk factor and PTSD would be greater among subjects encountering lower magnitude stressors relative to subjects encountering higher magnitude stressors. Furthermore, the background-foreground inversion hypothesis might hold for some stressors or some risk factors, but not for others. Regardless of the outcome, the findings would illuminate the etiology of PTSD.

Finally, one can vary the risk factor involved. In addition to intelligence, researchers might test this hypothesis by examining neuroticism, hippocampal volume, social support, and other variables that affect the likelihood of PTSD following exposure to trauma.

In conclusion, studies showing that people exposed to non-Criterion A events or events that would not have qualified as Criterion A stressors prior to the post-DSM-III expansion in the concept of trauma run counter to the assumption that only catastrophic trauma can produce the symptomatic profile of PTSD. If these people carry an especially heavy burden of vulnerability, then this would 
explain how "subtraumatic" stressors can sometimes produce a posttraumatic syndrome. 


\section{References}

1. American Psychiatric Association. Diagnostic and statistical manual of mental disorders - text revision. 4th ed. Washington, DC: American Psychiatric Association; 2000. 2. American Psychiatric Association. Diagnostic and statistical manual of mental disorders. 3rd ed. Washington, DC: American Psychiatric Association; 1980.

3. McNally RJ. Progress and controversy in the study of posttraumatic stress disorder. Annu Rev Psychol 2003;54:229252.

4. McNally RJ, Breslau N. Does virtual trauma cause posttraumatic stress disorder? Am Psychol 2008;63:282-283. 5. Schlenger WE, Caddell JM, Ebert L, et al. Psychological reactions to terrorist attacks: Findings from the National Study of Americans' Reactions to September 11. J Am Med Assoc $2002 ; 288: 581-588$

6. Dohrenwend BP. Toward a typology of high-risk major stressful events and situations in posttraumatic stress disorder and related psychopathology. Psychol Inj Law $2010 ; 3: 89-99$.

7. Gold SD, Marx BP, Soler-Baillo JM, Sloan DM. Is life stress more traumatic than traumatic stress? J Anx Dis $2005 ; 19: 687-698$ 
8. Long ME, Elhai JD, Schweinle A et al. Differences in posttraumatic stress disorder diagnostic rates and symptom severity between Criterion Al and non-Criterion Al stressors. J Anx Dis 2008;22:1255-1263.

9. Mol SSL, Arntz A, Metsemakers JFM, et al. Symptoms of post-traumatic stress disorder after non-traumatic events: Evidence from an open population study. Brit J Psychiat $2005 ; 186: 494-499$

10. Van Hooff M, MCFarlane AC, Baur J, Abraham M, Barnes DJ The stressor Criterion-A1 and PTSD: A matter of opinion? J Anx Dis $2009 ; 23: 77-86$.

11. Anders SL, Frazier PA, Frankfurt SB Variations in Criterion A and PTSD rates in a community sample of women. J Anx Dis 2011;25:176-184.

12. Engelhard IM, van den Hout MA, Weerts J, et al. Deployment-related stress and trauma in Dutch soldiers returning from Iraq: Prospective study. Brit J Psychiat $2007 ; 191: 140-145$.

13. Breslau N, Davis GC, Andreski P, Peterson E. Traumatic events and posttraumatic stress disorder in an urban population of young adults. Arch Gen Psychiatry 1991;48:216222. 
14. Yehuda R. Risk factors for posttraumatic stress disorder. Washington, DC: American Psychiatric Press; 1999. 15. MCNally RJ, Shin LM. Association of intelligence with posttraumatic stress disorder symptoms in Vietnam combat veterans. Am J Psychiatry 1995;152:936-938. 16. Macklin ML, Metzger LJ, Litz BT, et al. Lower precombat intelligence is a risk factor for posttraumatic stress disorder. J Consult Clin Psychol 1998;66:323-326. 17. Kaplan Z, Weiser M, Reichenberg A, et al. Motivation to serve in the military influences vulnerability to future posttraumatic stress disorder. Psychiatry Res 2002;109:45-49. 18. Breslau N, Lucia VC, Alvarado GF. Intelligence and other predisposing factors in exposure to trauma and posttraumatic stress disorder: A follow-up study at age 17 years. Arch Gen Psychiatry $2006 ; 63: 1238-1245$.

19. McNally RJ. Can we fix PTSD in DSM-V? Depress Anxiety $2009 ; 26: 597-600$

20. Breslau N. Posttraumatic syndromes and the problem of heterogeneity. East Lansing, MI: Department of Epidemiology, Michigan State University; 2010.

21. Helzer J. Methodological issues in the interpretation of the consequences of extreme situations. In: Dohrenwend BS, 
Dohrenwend BP, eds. Stressful life events and their contexts. New York, NY: Prodist; 1981: 108-129.

22. Breslau N, Chilcoat HD, Kessler RC, Peterson EL, Lucia VC. Vulnerability to assaultive violence: Further specification of the sex difference in post-traumatic stress disorder. Psychol Med 1999;29:813-821.

23. Ozer EJ, Best SR, Lipsey TL, Weiss DS. Predictors of posttraumatic stress disorder and symptoms in adults: A metaanalysis Psychol Bull 2003;129:52-73.

24. Schooler JW, Ambadar Z. Abuse Recollection

Questionnaire. Pittsburg, PA: Department of Psychology, University of Pittsburgh; 1997.

25. Weathers FW, Litz BT, Huska JA, Keane TM. The PTSD Checklist Civilian version. Boston: National Center for PTSD, Behavioral Science Division.

26. Terhakopian A, Sinaii N, Engel CC, Schnurr PP, Hoge CW. Estimating population prevalence of posttraumatic stress disorder: An example using the PTSD Checklist. J Trauma Stress $2008 ; 21: 1-11$.

27. Beck AT, Steer RA, Brown GK. Manual for the Beck Depression Inventory-II. San Antonio, TX: Psychological Corporation; 1996. 
28. Zachary RA. Shipley Institute of Living Scale (Revised). Los Angeles: Western Psychological Services; 1991. 29. Zachary RA, Crumpton E, Spiegel DE. Estimating WAIS-R from the Shipley Institute of Living Scale. J Clin Psychol $1985 ; 41: 532-540$. 30. March JS. The nosology of posttraumatic stress disorder. J Anx Dis 1990;4:61-82. 
Table 1

Means and Standard Deviations on Age and Questionnaire Measures as a Function of Stressor Severity

$$
\text { Severity Group }
$$

\begin{tabular}{lllllll} 
Measure & \multicolumn{1}{l}{ Low } & Medium & & High & F & P \\
Age & 36.1 & 42.4 & & 45.3 & 3.007 & .054 \\
& $(11.7)$ & $(12.2)$ & $(11.2)$ & & \\
IQ & 98.7 & 93.8 & 97.6 & 1.037 & .359 \\
PCL-C & $(13.9)$ & $(13.7)$ & $(16.3)$ & & \\
& $(13.0$ & 42.3 & $(16.1)$ & $(18.4)$ & & \\
BDI-II & 15.3 & 17.9 & $(12.1)$ & $(12.5)$ & &
\end{tabular}

Note. Standard deviations are in parentheses. IQ $=$ WAIS-R estimate from the Shipley Institute for Living Scale. PCL-C = Posttraumatic Stress Checklist-Civilian version. BDI-II = Beck Depression Inventory, second edition. 